\title{
Aprendizaje de las construcciones euclidianas con GeoGebra: elementos de una actividad formativa para futuros profesores de matemáticas
}

\author{
Juan Luis Prieto G., ${ }^{1,2}$ \\ juanl.prietog@gmail.com \\ https://orcid.org/0000-0003-0798-5191 \\ Elizabeth-H. Arredondo ${ }^{1}$ \\ Elizabeth.hernandez@ulagos.cl \\ https://orcid.org/0000-0002-5285-1603 \\ ${ }^{1}$ Universidad de Los Lagos (ULAGOS, Chile) \\ ${ }^{2}$ Asociación Aprender en Red (Venezuela)
}

Recibido: 2020-07-30; Aceptado: 2020-11-27

\begin{abstract}
Resumen
El trabajo describe los elementos constituyentes de una actividad formativa diseñada para promover aprendizaje sobre las construcciones euclidianas con GeoGebra por futuros profesores de matemáticas. Comenzamos fijando posición respecto al aprendizaje de dicho saber, partiendo de la concepción histórico-cultural de aprendizaje como procesos de objetivación. Luego conceptualizamos el saber sobre las construcciones euclidianas con GeoGebra como un saber docente que se nutre desde lo disciplinario. Los elementos de la actividad incluyen el motivo, objetivo y metas de la formación, así como las tareas y su modo de implementación. Finalizamos con algunas consideraciones sobre la puesta en escena de la propuesta.

Palabras clave: Procesos de objetivación; Saberes docentes; Tareas de construcción; Formación inicial docente
\end{abstract}

\section{Aprendizagem das construções euclidianas com GeoGebra: Elementos de uma atividade formativa para futuros professores de matemáticas}

\section{Resumo}

O presente trabalho descreve os elementos que constituem uma atividade formativa criada para promover a aprendizagem sobre as construções euclidianas com GeoGebra por futuros professores de matemáticas. Começamos fixando posição ao respeito da aprendizagem do referido saber, baseados em uma concepção histórico-cultural da aprendizagem como processo de objetivação. Logo se faz uma conceptualização do saber sobre as construções euclidianas com GeoGebra como um saber docente que se nutre desde o tópico disciplinar. Os elementos da atividade incluem o motivo, objetivo e metas da formação, assim como das tarefas e o modo de implementação. Finalizamos com algumas considerações sobre a aplicação da proposta.

Palavras chaves: Processo de Objetivação, Saberes docentes, Tarefas de construção; Formação inicial do docente 


\title{
Learning of Euclidian constructions with GeoGebra: elements of a training activity for preservice mathematics teachers
}

\begin{abstract}
The paper describes the constituent elements of a formation activity designed to promote learning about Euclidean constructions with GeoGebra by preservice mathematics teachers. We begin by establishing a position with respect to the learning of this knowledge, starting from the historical-cultural conception of learning as processes of objectivation. Then we conceptualize knowledge about Euclidean constructions with GeoGebra as teaching knowledge that is nourished from the disciplinary point of view. The elements of the activity include the motive, objective and goals of the training, as well as the tasks and their mode of implementation. We end with some considerations about the staging of the proposal.

Keywords: Objectification processes; Teaching knowledge; Construction tasks; Initial teacher training
\end{abstract}

\section{Introducción}

La formación de profesores de matemáticas, como área de investigación y práctica educativa, procura la mejora de los procesos de aprendizaje y desarrollo profesional del profesorado en todas sus etapas o fases (inicial, principiante y continua), por medio de actividades formativas diversas y dinámicas (Krainer y Llinares; 2010).

En la etapa de formación inicial, los temas de investigación incluyen especialmente lo relativo a las capacidades de los futuros profesores para su desempeño en la práctica profesional; capacidades éstas que pueden comprometer la calidad de los programas de formación docente en las universidades. En las últimas décadas, este se ha hecho notar gracias al esfuerzo de los investigadores tanto por comprender la naturaleza, origen, fuentes y modos de producción del saber que sustenta el acto de enseñar matemáticas (Depaepe et al., 2013; Neubrand, 2018; Valente, 2017), como por producir dispositivos y modos de usar estos dispositivos en la formación del profesorado para favorecer el aprendizaje del saber docente (Llinares, 2019a; Tirosh y Wood, 2008). Sobre los dispositivos de aprendizaje (actividades, entornos, lecciones, cursos, etc.), vale destacar que su diseño ha ganado importancia en la formación inicial debido a las posibilidades que ofrecen de incorporar distintos artefactos materiales y simbólicos a la dinámica formativa, atendiendo a los principios teóricos sobre el aprendizaje que orienten su diseño y creando así las condiciones necesarias para el encuentro de los futuros profesores con el saber docente (Llinares, 2004). 
Reconociendo el auge de los dispositivos de aprendizaje en la formación inicial de profesores de matemáticas, el presente trabajo caracteriza una actividad formativa en geometría cuyo diseño está fundamentado en una perspectiva histórico-cultural del aprendizaje (Radford, 2020) que reconoce en la actividad humana el vínculo natural que puede conectar a los futuros profesores con el mundo de la enseñanza. En la formación inicial, esta conexión no siempre ocurre (al menos de forma explícita) en los cursos centrados en las disciplinas científicas (p. ej., la geometría euclidiana), pese a que los futuros profesores aprenden las matemáticas escolares al mismo tiempo que se van constituyendo en sujetos docentes (Fiorentini, 2005; Tardif, 2002).

Como alternativa, la actividad formativa que se presenta busca promover el aprendizaje de un saber docente en geometría referido a las construcciones euclidianas ${ }^{1}$ producidas con el software GeoGebra, el cual se corresponde con la enseñanza como una profesión. Este trabajo forma parte de un proyecto de investigación doctoral que tiene por objetivo caracterizar el aprendizaje de este saber por parte de futuros profesores de matemáticas en los últimos años de su carrera universitaria.

El trabajo se estructura en cinco apartados que comienzan con posicionamientos sobre el aprendizaje de saberes docentes en geometría como procesos de objetivación y sobre el saber acerca de las construcciones euclidianas con GeoGebra como saber docente. Estos primeros apartados ofrecen el referencial teórico necesario para orientar el diseño de la actividad formativa. Posteriormente, el trabajo presenta los elementos que conforman la actividad formativa y describe cómo ha de ser su despliegue en un contexto de formación inicial de profesores de matemáticas. Por último, se plantean algunas consideraciones finales relacionadas con el diseño.

\section{Aprendizaje como procesos de objetivación}

En este trabajo, el aprendizaje de un saber docente en geometría en contextos de formación inicial de profesores de matemáticas es interpretado a la luz de la concepción histórico-cultural de aprendizaje que aporta la Teoría de la Objetivación (TO) (Radford, 2013; 2014a). En general, la TO concibe el aprendizaje como el encuentro de los individuos con el saber histórico y cultural (p. ej., con el saber matemático, pedagógico, estético, entre otros); un saber que, en

\footnotetext{
${ }^{1}$ Conjunto de problemas de construcción con regla y compas que pueden encontrarse en los Elementos de Euclides y que siguen vigentes en el currículo de matemáticas de la Educación Básica y Media en Chile.
} 
determinado momento de la vida, objeta al individuo, es decir, le opone resistencia. Una definición más operativa del aprendizaje en la TO le considera como procesos de objetivación, esto es, como procesos sociales de:

[...] toma de conciencia progresiva y crítica, de un sistema de pensamiento y acción cultural e históricamente constituido, sistema que gradualmente notamos, y que al mismo tiempo dotamos de sentido. Los procesos de objetivación son aquellos procesos de notar algo culturalmente significativo, algo que se revela a la conciencia [...] por medio de la actividad corpórea, sensible, afectiva, emocional, artefactual y semiótica. (Radford, 2020, p. 20)

A partir de esta definición, es posible entender los procesos de objetivación que ocurren en instituciones de formación del profesorado como actos colectivos y progresivos de toma de conciencia de aquello que se constituye en saber docente en el decurso de una actividad formativa concreta que es, además, una actividad social, corpórea, sensorial y artefactual. En esta conceptualización del aprendizaje subyacen tres ideas fundamentales para el desarrollo de este trabajo: saber, toma de conciencia y actividad.

En cuanto al saber, la TO lo define como una síntesis dinámica y evolutiva de expresión, acción y pensamiento, constituida histórica y culturalmente (Radford, 2017a). De ahí que el saber se constituya a partir de laboriosos e inacabados procesos históricos de codificación y refinamiento de determinadas formas de expresión, acción y pensamiento humano. Según Radford (2017a), para que el saber pueda emerger a la realidad concreta, éste debe ponerse en movimiento y tomar la forma de un conocimiento. Acerca del conocimiento, éste es concebido por el autor como "el contenido conceptual concreto en el que se manifiesta o actualiza o materializa o encarna el saber. Su contenido conceptual concreto aparece y puede aparecer únicamente en una actividad [...]" (p. 109).

Desde la perspectiva de la TO, el saber docente puede entenderse como una síntesis dinámica de expresión, acción y pensamiento que la cultura escolar (o académica) ofrece al profesor (o futuro profesor) para reafirmarse (y constituirse) en su condición de docente a través de su trabajo. Que el saber docente sea una síntesis dinámica significa que éste (como todo saber cultural) se encuentra en movimiento constante, cambiando de cultura en cultura (Radford, 2014a). Vale recordar que el saber docente surge a partir de la necesidad social e histórica de "hacer de la práctica pedagógica y de la acción del profesor un trabajo basado en parámetros 
racionales, junto con la expectativa de obtener resultados predecibles e inmediatos de este trabajo" (Xavier, 2014, p. 831).

En cuanto a la especificidad del saber docente, Tardif (2002) explica que se trata de "un saber plural, formado por una amalgama, más o menos coherente, de saberes procedentes de la formación profesional y disciplinarios, curriculares y experienciales" (p. 29). Para el autor, este saber es movilizado por el profesor durante el trabajo con sus alumnos en el aula, y de manera más general, dentro de la escuela. Por saber profesional se entiende aquel saber producido por las ciencias de la educación (p. ej., la educación matemática) y difundido a través de las instituciones de formación del profesorado; mientras que por saber disciplinario se entiende aquel producido por la tradición cultural y seleccionado por las instituciones de formación para su difusión a través de cursos o asignaturas.

Con respecto a la toma de conciencia, la TO le considera como la forma específicamente humana en que cada individuo reconoce los objetos de la cultura (en particular, el saber) en la realidad concreta y se posiciona críticamente ante tales objetos. Para Radford (2006), tomar conciencia de un saber cultural implica una re-flexión, es decir, un movimiento dialéctico entre la realidad objetiva y el individuo "que la refracta (y la modifica) según las interpretaciones y sentidos subjetivos propios" (p. 108). Durante este movimiento, la conciencia se forma y se transforma, haciendo de la reflexión crítica un acto de producción de la propia conciencia.

En la formación inicial, la toma de conciencia de un saber docente puede entenderse como un acto subjetivo, emocional y afectivo de reflexión y discernimiento crítico del futuro profesor ante las formas de expresión, acción y pensamiento que constituyen dicho saber. En este acto se forman "sensibilidades culturales para ponderar, reflexionar, comprender, disentir, objetar y sentir a los otros, a nosotros mismos y a nuestro mundo" (Radford, 2017b, p. 122). Estas sensibilidades son las que permiten al futuro profesor orientarse en la formación, por ejemplo, al producir construcciones euclidianas con GeoGebra o involucrarse responsablemente en las experiencias de producción de estas construcciones hechas por otros compañeros. 
Aprendizaje de las construcciones euclidianas con GeoGebra: elementos de una actividad formativa...

Finalmente, para que el saber se convierta en objeto de conciencia, es necesario que una actividad sensual $^{2}$ y práctica le ponga en movimiento (Radford, 2020). En la TO, la actividad es la categoría ontológica y epistemológica fundamental para entender el modo en que los individuos se expresan, actúan y piensan en su cultura. Para evitar confusiones con otros significados de la actividad que le alejan de su carácter de espacio de producción humana, Radford (2017b) se refiere a ella como labor conjunta, esto es, como "una forma de vida, algo orgánico y sistémico, un evento creado por una búsqueda común -es decir una búsqueda con otros-de la solución a un problema planteado, búsqueda que es al mismo tiempo cognitiva, emocional y ética" (p. 125). Esta definición pone de manifiesto que la enseñanza y el aprendizaje no son dos actividades separadas (una realizada por el profesor y otra por el alumno), sino "una misma y única actividad: la labor conjunta de los profesores y los estudiantes" (Radford, 2020, p. 24).

Teniendo en cuenta lo anterior, en este trabajo se ha empleado el término actividad formativa para indicar esa forma social, corpórea, sensorial y artefactual de labor conjunta mediante la cual los futuros profesores y su formador se implican mutuamente en la búsqueda de respuestas a los problemas que orientan sus acciones para el logro de unas metas. Esta búsqueda hace posible la toma de conciencia progresiva del saber docente puesto en juego. Es decir, la actividad formativa puede transformar las condiciones iniciales de una realidad (p. ej., aquella que ofrece la respuesta individual de un futuro profesor a la tarea de construir un triángulo con GeoGebra) y, con ello, hacer que el saber aparezca "de manera muy similar, y con la fuerza estética semejante, a la manera como la música aparece fonéticamente en una sala de conciertos" (Radford, 2018, p. 98).

El producto de la labor conjunta constituye la obra común, esto es, "la apariencia sensual del saber" (Radford, 2018, p. 98). Por lo tanto, se puede afirmar que la obra común producida por la actividad formativa de los futuros profesores y su formador es tanto aquella forma material del saber docente en la realidad concreta como el reflejo de tal saber en la conciencia de cada individuo que participa en su producción. Sobre esto último, Leontiev (1978, p. 123) afirmaba que "la actividad del hombre es lo que constituye la sustancia de su conciencia".

\footnotetext{
${ }^{2}$ Según Fischbach $(2015$, p. 31), "Las facultades sensibles [humanas] como el oído o la vista no permanecen idénticas eternamente, sino que se producen, resultan y son modificadas por la actividad humana, participan en esta actividad y son un aspecto de ella".
} 
Un aspecto que la TO destaca de toda labor conjunta se refiere a los recursos semióticos empleados por los individuos para la consecución de la obra común. Estos recursos son consustanciales al pensamiento humano y se materializan a través del cuerpo (acciones kinestésicas, gestos y sensaciones), del uso de signos (lenguaje natural o simbólico) y de artefactos culturales (materiales concretos) (Radford et al., 2009). Respecto a los artefactos culturales, vale decir que éstos no son neutrales. Por el contrario, al ser inseparables de la actividad humana, los artefactos portan significados históricos y culturales que afectan inevitablemente las formas en que los individuos se expresan, actúan y piensan durante la actividad (Radford, 2014b).

\section{Saber acerca de las construcciones euclidianas con GeoGebra}

La actividad formativa pone en movimiento un saber específico de la enseñanza de la geometría que, en este trabajo, se ha convenido en llamar: saber acerca de las construcciones euclidianas con GeoGebra (se abrevia SACEG). Tratándose de un saber docente, el SACEG se encuentra enraizado en la labor que realizan los profesores de matemáticas de educación básica y media de Chile, particularmente cuando trabajan con sus alumnos las construcciones euclidianas producidas con el software GeoGebra. En la práctica, las fuentes del SACEG son diversas y pueden incluir saberes disciplinarios, profesionales y experienciales de los profesores. Seguidamente se presenta cómo los saberes disciplinarios alimentan al SACEG y contribuyen con su conceptualización.

\section{La vertiente disciplinaria del SACEG}

Como su nombre indica, el SACEG se nutre de la geometría euclidiana como una disciplina científica integrada en la formación inicial de profesores de matemáticas. Siguiendo a Valente (2017), esta disciplina científica aporta al profesor esa geometría que es su objeto de enseñanza, es decir, aquello que se debe enseñar. Por lo tanto, la vertiente disciplinaria del SACEG comprende las síntesis de expresión, acción y pensamiento acerca de los objetos de la teoría elemental de la geometría plana (p. ej., rectas, ángulos, triángulos, cuadriláteros), que se encuentran codificadas en la cultura escolar como procedimientos de construcción de estos objetos con determinados artefactos. 
Aprendizaje de las construcciones euclidianas con GeoGebra: elementos de una actividad formativa...

Las raíces del SACEG, en lo disciplinario, pueden encontrarse en el periodo de la Grecia clásica (450-300 a. de C.), cuando la producción de tratados sobre los fundamentos de la geometría era parte de una larga tradición de abstracción y sistematización progresiva de todo lo creado por el orden científico, desde Hipócrates de Quíos hasta Euclides (Ríbnikov, 1987; Scriba y Schreiber, 2015, Vega-Reñon, 1990).

En este periodo se establecen las situaciones o problemas elementales de la geometría que otorgan sentido al SACEG, en particular las construcciones con regla y compás. Mediante estos problemas, los matemáticos griegos demostraban la existencia de un objeto geométrico con ciertas propiedades (objeto deseado), partiendo de unas condiciones iniciales (objetos dados) (Rey y Babini, 1985). El método para resolver estos problemas consistía básicamente en describir el procedimiento de construcción del objeto deseado, usando solo regla y compás (artefactos), y probar que tal procedimiento entregaba el objeto con las propiedades deseadas (Scriba y Schreiber, 2015). Tal método revela una forma prototípica o ideal de expresión, acción y pensamiento acerca de los problemas de construcción, característica de la sociedad griega, la cual fue adoptada por Euclides en su famoso tratado de geometría sintética y teoría de los números, llamado Elementos.

Por medio de la resolución de problemas de construcción con regla y compás (producción del procedimiento de construcción y su prueba), los matemáticos griegos ponían en movimiento esas síntesis de expresión, acción y pensamiento geométrico de la época, dentro de una súper estructura simbólica o modo de investigar sobre los objetos de la geometría altamente sofisticada, la cual se resume en el método axiomático-deductivo de Euclides (Ríbnikov, 1987). Este método no ha estado exento de contradicciones internas. Entre las objeciones al método se encuentra el uso recurrente de postulados tácitos en la solución de los problemas (Boyer, 1987). Las contradicciones que afectaban al sistema axiomático-deductivo de Euclides motivaron el avance de la geometría entre los siglos XVII y XX, produciéndose transformaciones en su naturaleza y modos de producción.

En la actualidad, el SACEG se alimenta de sofisticadas y evolucionadas definiciones y axiomas de la geometría elemental, de algo más que el método sintético euclídeo para explorar esta geometría (p. ej., el método analítico originado en el siglo XVII) y con artefactos digitales que hacen posible pensar geométricamente de formas novedosas en el aula. Según Laborde 
(1997), hoy en día las construcciones euclidianas elaboradas con software dinámico (un artefacto digital) comprenden actividades de enseñanza y aprendizaje de la geometría propicias para el reconocimiento de vínculos entre las evidencias visuales que aportan los dibujos en la pantalla (invarianza de las propiedades espaciales del dibujo) y los hechos geométricos (elementos del sistema axiomático-deductivo de la geometría euclidiana), condicionados por la conceptualidad detrás de las herramientas de construcción que ofrece el software.

Siguiendo las ideas de Radford (2014b), el software GeoGebra representa un artefacto cultural (de naturaleza digital) que proporciona a los usuarios tanto una serie de contenidos conceptuales (herramientas de construcción, medida y otras opciones) como un espacio de trabajo estructurado conceptualmente (apariencias del software) para que ellos experimenten con tales contenidos y produzcan formas novedosas de construir los dibujos dinámicos ${ }^{3}$ y validar estas construcciones. Por ejemplo, si se desea dibujar un triángulo rectángulo con GeoGebra, la herramienta Polígono sugiere un procedimiento de construcción del objeto que demanda informar al software cuáles son los vértices que le definen (Figura 1).

Figura 1. Conceptualización del polígono por la herramienta correspondiente

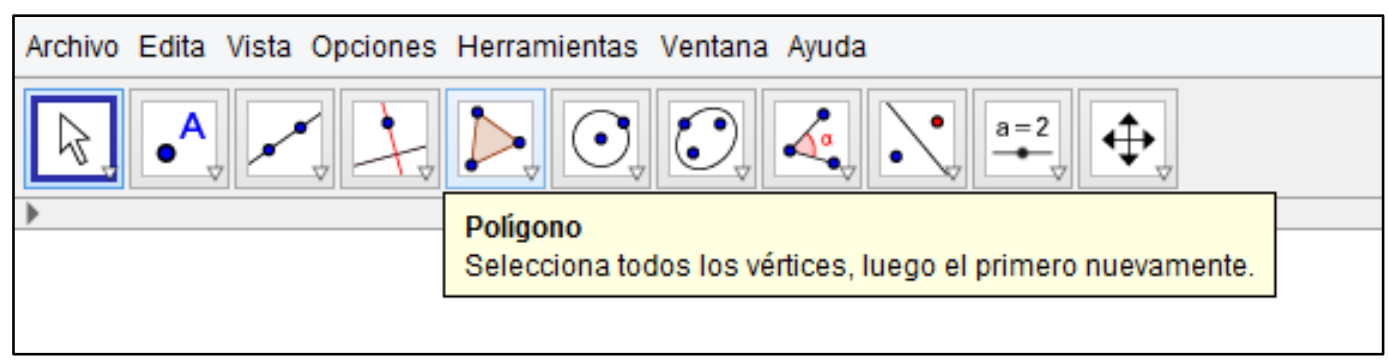

Fuente: Autores

La incorporación de esta clase de artefactos digitales en la actividad del aula ha afectado la manera en que profesores y alumnos se expresan, actúan y piensan sobre los objetos de la geometría y su existencia en la realidad escolar como objetos materiales (como dibujos dinámicos en la pantalla del computador) (Laborde et al., 2006). Por ejemplo, en actividades mediadas por software dinámico, los objetos geométricos son vistos como entidades cuyo comportamiento puede ser investigado y descrito, por ejemplo, a través de la modalidad de arrastre que ofrece el software, favoreciendo con ello el reconocimiento de propiedades

\footnotetext{
${ }^{3}$ Por dibujo dinámico se entiende aquel dibujo creado con algún software dinámico, de manera que "[...] conserve ciertas propiedades espaciales impuestas cuando se desplace por uno de los puntos básicos del dibujo." (Laborde, 1997, p. 42).
} 
Aprendizaje de las construcciones euclidianas con GeoGebra: elementos de una actividad formativa...

espaciales del dibujo dinámico que se mantienen invariantes en el movimiento (Sinclair y Robutti, 2013). Según Arzarello, et al. (2002), la modalidad de arrastre consiste en:

(...) mover un punto libre o semi-libre [dependiente] para ver si el dibujo mantiene las propiedades iniciales. Si esto es así, entonces la figura pasa la prueba; en caso contrario, el dibujo no fue construido de acuerdo con las propiedades geométricas que se quería que tuviese. (p. 67)

Con respecto a la prueba matemática, aunque ésta se entienda en lo disciplinario como una cadena formal de razonamientos que parte de unos axiomas y evoluciona hacia la conclusión mediante pasos lógicos, la llegada del software dinámico al aula ha generado la aparición de nuevas formas de establecer la verdad de un hecho geométrico (De Villiers, 1998). En este trabajo, la prueba matemática se entiende como ese proceso social, corporal, simbólico y artefactual de producción de formas de argumentación para explicar, justificar o validar la consistencia geométrica de una construcción realizada con GeoGebra, con miras a convencer a alguien de la validez de ese conocimiento (Fiallo, 2011).

La conceptualización del SACEG desde la vertiente disciplinaria pone de manifiesto la importancia de la actividad de resolución de tareas de construcción euclidianas con GeoGebra como la fuente que actualiza o materializa este saber en la formación. Por tanto, es posible concluir que el SACEG se expresa en la capacidad potencial de los futuros profesores para (i) construir un dibujo dinámico en la pantalla del software que represente al objeto geométrico demandado por la tarea, (ii) comunicar (de manera oral y/o escrita) el procedimiento de construcción del dibujo dinámico en función de pasos y acciones, y (iii) probar la validez o consistencia geométrica del dibujo dinámico producido, atendiendo a las condiciones impuestas por la tarea correspondiente.

\section{Elementos de la actividad formativa}

La actividad formativa produce experiencias de vida únicas e irrepetibles que revelan el modo en que los futuros profesores y su formador toman conciencia del SACEG de forma progresiva y crítica. De ahí la importancia de develar sus elementos. En principio, la TO considera al motivo, objetivo, metas y tareas como elementos que estructuran la labor conjunta del aula de matemáticas (Radford, 2017b). Sin embargo, estos elementos se complementan con una visión dialéctico-materialista de la manera en que la actividad es realizada, es decir, de 
aquello que Radford (2014a; 2020) denomina ejes de la actividad: (i) formas de producción de saberes, y (ii) formas de colaboración humana. Son estos ejes los que permiten considerar el contenido y las interacciones entre los sujetos como elementos propios de la actividad formativa.

Según Leontiev (1978), toda actividad humana responde a una necesidad material o espiritual de los individuos que se involucran en ella, siendo ésta objetivada en el motivo que impulsa esa labor. Este motivo se encuentra estrechamente ligado a un objeto (material o ideal) que le confiere determinada orientación a la actividad. En palabras de Reverand (2004, p. 165), el objeto "constituye el resultado futuro, anticipado de la actividad, todo aquello que el hombre desea alcanzar, obtener, crear”. En consecuencia, la obra común puede entenderse como esa forma material que adquiere el objeto de una actividad en la realidad, luego que ésta es realizada.

Finalmente, ya que "una actividad que se desenvuelve en alguna medida presupone el logro de una serie de fines concretos" (Leontiev, 1978, p. 84), para que el trabajo conjunto se mueva en la dirección de su objeto, conviene identificar una o más metas que puedan lograrse a través de tareas específicas (Radford, 2017b). La meta es la representación consciente del resultado al que se subordina alguna acción o cadena de acciones, mientras que la tarea constituye una meta que se estipula bajo ciertas condiciones.

\section{Motivo, objeto y metas}

Una necesidad latente en las instituciones de formación de profesores de matemáticas es lograr que estos sujetos reconozcan el potencial de las tareas propuestas a sus alumnos para desarrollar formas de pensar y actuar matemáticamente que se consideran importantes para el funcionamiento de la sociedad actual (Linares et al., 2008). De acuerdo con esta necesidad, el motivo de la actividad formativa propuesta es analizar el potencial de las tareas de construcción euclidiana con GeoGebra para promover discursos y prácticas de construcción, comunicación y razonamiento característicos de la geometría euclidiana.

De lo anterior se tiene que el objeto de la actividad formativa es el encuentro progresivo y crítico con el SACEG desde su vertiente disciplinaria. En otras palabras, se espera que la actividad formativa resulte en la toma de conciencia del futuro profesor de aquello que se constituye un SACEG para la producción de la obra común, esto es, en la resolución de tareas 
de construcción euclidiana con GeoGebra. Para que la labor conjunta se despliegue en la dirección del objeto, se han formulado las siguientes metas:

1. Producir construcciones euclidianas con GeoGebra (dibujos dinámicos) que den respuesta a las exigencias de las tareas que le demandan.

2. Comunicar (en forma oral y/o escrita) los procedimientos de construcción de los dibujos dinámicos.

3. Probar que los procedimientos de construcción de los dibujos dinámicos son consistentes desde el punto de vista geométrico.

\section{Tareas}

En este trabajo, las tareas se conciben como parte consustancial de la actividad formativa en cuanto aportan las condiciones iniciales para que los futuros profesores configuren su experiencia con el saber puesto en movimiento y comprendan la naturaleza del trabajo docente en geometría (Watson y Ohtani, 2015). En una actividad formativa, la tarea tiene como propósito orientar las acciones de los futuros profesores hacia el logro de las metas.

Un total de 16 tareas de construcción euclidianas con GeoGebra fueron elaboradas teniendo en cuenta los siguientes aspectos:

\section{Tipo de tareas}

Según la clasificación de tareas geométricas en entornos dinámicos que propone Laborde (1997), las tareas diseñadas se corresponden, en principio, con las clásicas tareas de producción de Cabri-dibujos ${ }^{4}$, las cuales demandan la construcción de dibujos dinámicos representativos de objetos geométricos dados mediante descripción verbal. Según la autora, esta clase de tareas demanda la producción de:

(...) un dibujo en la pantalla que conserve ciertas propiedades espaciales impuestas cuando se desplace uno de los puntos básicos del dibujo. La tarea para el alumno consiste, por tanto, en elaborar un procedimiento

\footnotetext{
${ }^{4}$ Un Cabri-dibujo es un dibujo dinámico creado con el software Cabri- Géomètre. Vale destacar que esta clase de dibujos también puede reproducirse con otras aplicaciones con las características de un software de geometría dinámica, como es el caso de GeoGebra, un software de Matemática Dinámica completamente gratuito y accesible desde la Web.
} 
de producción del Cabri-dibujo, basado en las primitivas geométricas disponibles. (p. 42)

\section{Contenido conceptual de las tareas}

El contenido conceptual específico de las tareas de construcción euclidianas con GeoGebra fue definido a partir de la conceptualización del SACEG (descrita en un apartado anterior) y del análisis de algunas fuentes documentales históricas (p. ej., Rey y Babini, 1985, Ribnikov, 1987; Scriba y Schreiber, 2015; Vega-Reñon, 1990). De esta manera, ha sido posible identificar: (i) las construcciones con regla y compás que dan lugar a las tareas de la actividad formativa, (ii) la solución de estos problemas que se sugieren en algunos textos de geometría, y (iii) las categorías conceptuales y sistemas semióticos de significación puestos en juego durante la resolución. Toda esta información aporta la materia prima para la elaboración de las tareas de construcción euclidianas con GeoGebra y algunas respuestas esperadas.

En la Tabla 1 se muestra el conjunto de proposiciones o problemas de construcción con regla y compás que han servido para la elaboración de las tareas. Tales proposiciones se refieren a la construcción de figuras planas y la división de segmentos en una razón dada, y fueron seleccionadas a partir de una revisión hecha a los seis primeros libros de los Elementos de Euclides en español ${ }^{5}$ y de la obra Compendio Mathematico de Tosca (1757). La selección de las proposiciones se hizo a partir de la relevancia histórica del problema en función de su presencia directa o indirecta en el currículo de matemáticas chileno.

Tabla 1. Proposiciones seleccionadas para la elaboración de las tareas

\begin{tabular}{lcl}
\hline \multicolumn{1}{c}{ Fuente } & Libro & \multicolumn{1}{c}{ Proposición(es) } \\
\hline Euclides (1991, Libros I-IV) & I & I.1, I.9, I.10, I.12 \\
Euclides (1994, Libros V-IX) & II & II.11 \\
Simson (1774, Libros I-VI) & IV & IV.10 \\
& VI & VI.9, VI.11, VI.12*, VI.13, VI.16*, VI.30 \\
Tosca (1757, Tratado III, Tomo I) & I & I.9 \\
& II & II.1, II.2, II.4 \\
& VI & VI.5 \\
\hline
\end{tabular}

(*) Estas dos proposiciones dieron lugar a una misma tarea de construcción. Fuente: Autores

La revisión de las obras consultadas también proporcionó información acerca de la solución de cada proposición, la cual estaba comprendida de un procedimiento de construcción con regla y compás, el dibujo geométrico asociado y la prueba de la validez de la construcción.

\footnotetext{
${ }^{5}$ Se revisaron las traducciones de Roberto Simson (2014) y Maria Luisa Puertas Castaños (Euclides, 1991; 1994).
} 
Esta información se considera importante para el diseño de las tareas de construcción euclidiana con GeoGebra, ya que aporta los insumos necesarios para formular los enunciados y establecer algunas posibles respuestas.

Estos insumos fueron recabados y organizados en cuadros de doble entrada (Figura 2), en los cuales se incorporó también información sobre cada proposición tal como es tratada en textos de geometría ${ }^{6}$ recomendados en los cursos de geometría euclidiana de algunas carreras de Pedagogía en Matemáticas de Chile. Se recurrió a estas últimas fuentes, ya que era necesario contar con referencias actuales de la axiomática geométrica moderna que, como se sabe, constituye una forma actualizada y sofisticada de la axiomática de Euclides en los Elementos y forma parte de la preparación académica de los futuros profesores.

Figura 2. Cuadro usado para recabar información acerca de la solución de la proposición I.9

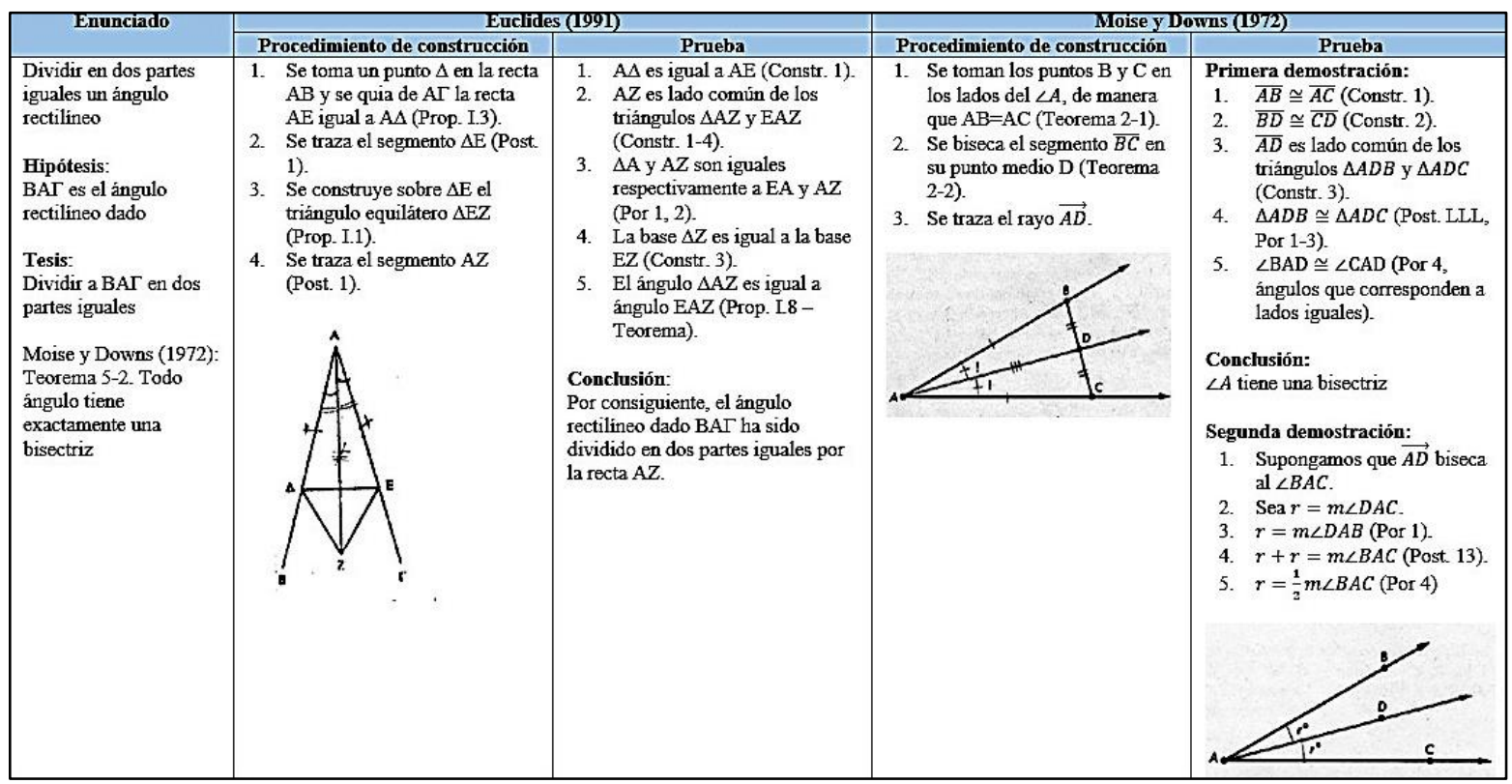

Fuente: Autores

La información recabada en los cuadros pone de manifiesto los conceptos, propiedades y relaciones geométricas (categorías conceptuales) que son evocados en las respuestas sugeridas en los textos, así como algunos procedimientos, signos y artefactos (sistemas semióticos) codificados históricamente que aseguran la sostenibilidad del saber acerca de las construcciones euclidianas y se ofrecen a los individuos para expresarse, actuar y pensar sobre las categorías conceptuales.

${ }^{6}$ Por ejemplo, la obra Geometría Moderna de Moise y Downs (1972). 
Por ejemplo, la información extraída de la proposición I.9 (Figura 2) da cuenta de algunos procedimientos prototípicos o ideales de resolución del problema de dividir un ángulo dado en dos partes iguales, utilizando regla y compás. En términos modernos, este problema equivale a la construcción de la bisectriz de un ángulo conocido. En estos procedimientos, un repertorio de signos (dibujos, notación simbólica y marcas) y artefactos (teoría geométrica ${ }^{7}$, lenguaje escrito e instrumentos de construcción), altamente sofisticados, entran en juego para dotar de significado la idea de bisectriz de un ángulo como aquel rayo que biseca al ángulo.

Tras definirse los aspectos anteriores, la elaboración de las tareas de la actividad formativa continuó con la transformación de cada proposición de la Tabla 1 en una tarea de construcción euclidiana con GeoGebra que incluía un enunciado, unas preguntas y una hoja de trabajo de GeoGebra (archivo .ggb) sobre la cual realizar la construcción. El enunciado de la tarea sigue la estructura de las proposiciones en los textos consultados (enunciado, hipótesis y tesis), reduciendo el caso general de la proposición a un caso particular cuyos elementos dados se muestran en la hoja de trabajo. Las preguntas están orientadas hacia el logro de las metas de la actividad formativa (construir, comunicar y probar). Así, por ejemplo, la Figura 3 muestra la tarea de construcción euclidiana GGB-4b que resulta de la transformación de la proposición II.2 de Tosca (1757).

\footnotetext{
${ }^{7}$ Postulados, definiciones, teoremas, etc.
} 
Figura 3. Ejemplo de una tarea de construcción euclidiana con GeoGebra

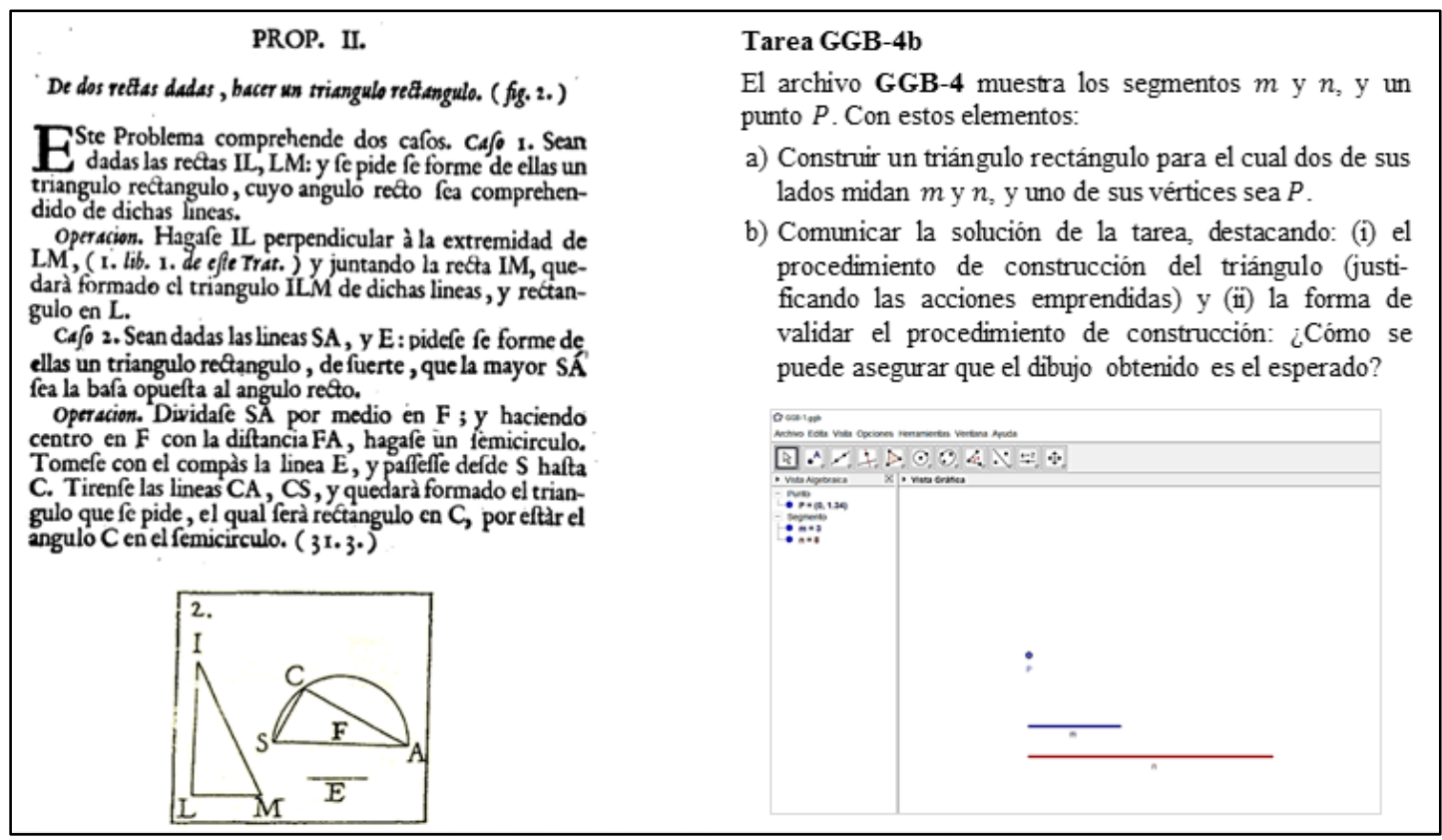

Fuente: Autores

Junto con la elaboración de las tareas, se establecieron las respuestas posibles que pudieran aparecer al ser aplicadas en la actividad formativa. En esta parte conviene resaltar que la aplicación de una tarea de construcción euclidiana con GeoGebra tiende a la producción de una forma de obra común que se puede considerar una respuesta adecuada a la tarea en la medida en que incluya: (i) el dibujo dinámico esperado, (ii) la comunicación (oral y/o escrita) del procedimiento de producción del dibujo dinámico, y (iii) la prueba de la validez o consistencia geométrica del dibujo, según las condiciones iniciales de la tarea.

Estas tres componentes de la respuesta fueron anticipadas por medio del análisis de la información correspondiente al postulado que origina la tarea (organizada con anterioridad). Por ejemplo, al analizar la información suministrada por la proposición II.2 que originó la tarea GGB-4b (Figura 3), se identificaron dos casos posibles de construcción del triángulo rectángulo según la posición que ocupan los objetos dados (lados del triángulo) en el dibujo. Claro está, la funcionalidad del software utilizado (como editor gráfico) y la variedad de contenidos conceptuales que éste ofrece hace posible que la resolución de una tarea de construcción euclidiana con GeoGebra sea una ocasión para ampliar el campo de experimentación posible con los dibujos dinámicos (Laborde, 1997). 
Sin embargo, la información de la proposición II.2 sugiere la posibilidad de que se produzca en la actividad formativa un dibujo dinámico similar al de la Figura 4, para el cual $m$ y $n$ son catetos del triángulo (caso 1 de la proposición II.2), así como también una descripción del procedimiento de construcción de ese triángulo con GeoGebra. Tal procedimiento es organizado en pasos y acciones, según la conceptualidad de la herramienta Polígono que es usada para producir el dibujo dinámico.

Figura 4. Respuesta posible a la tarea GGB-4b (dibujo dinámico y procedimiento de construcción)

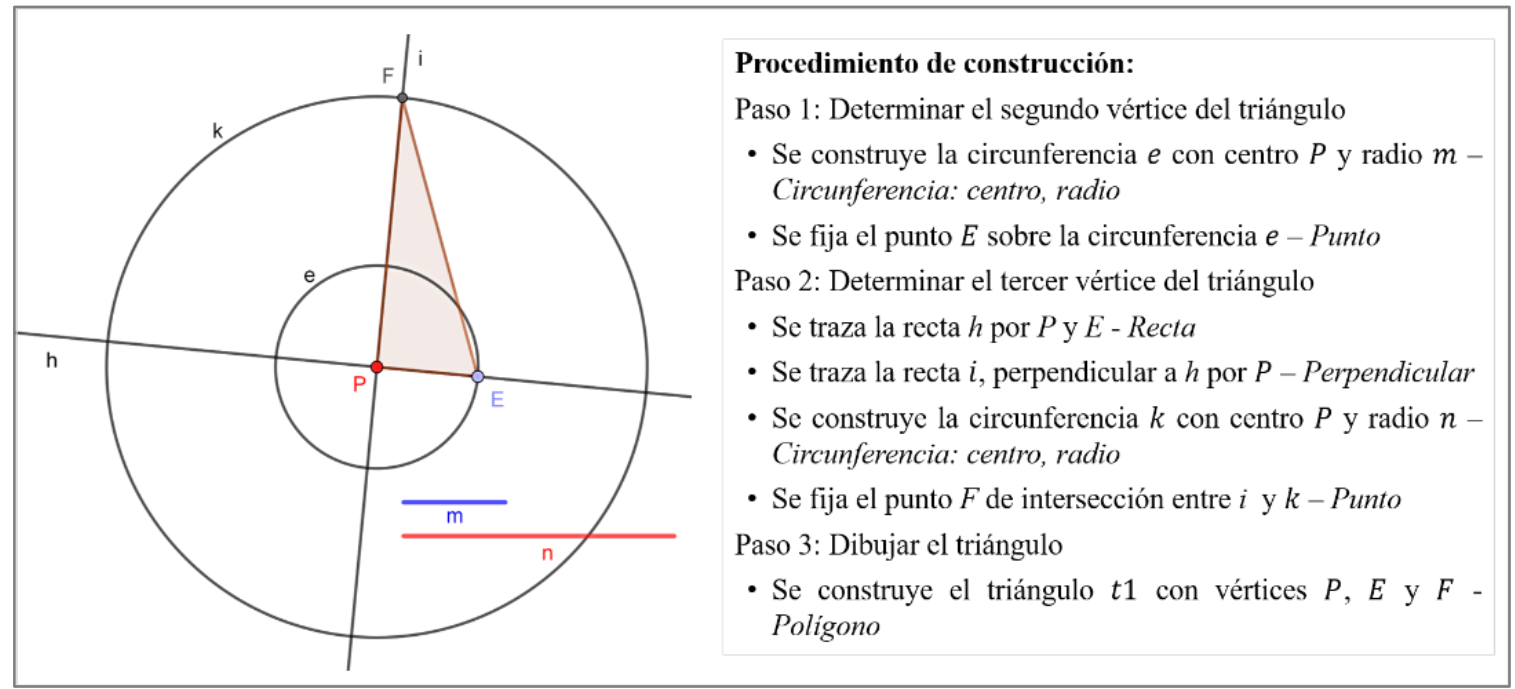

Fuente: Autores

En cuanto a la prueba, ésta ha de verse reflejada en las explicaciones que ofrecen los futuros profesores y el formador en cuanto a la consistencia geométrica de la construcción realizada, las cuales podrían estar basadas en las relaciones entre el comportamiento del dibujo dinámico en la pantalla y la teoría geométrica (Laborde, 1997). En este sentido, podría esperarse que las explicaciones den cuenta del porqué de la resistencia (o no) del dibujo dinámico a la prueba del arrastre, es decir, el motivo (o motivos) de mantenerse invariante (o no) de ciertas propiedades espaciales del dibujo tras el arrastre por alguno de sus elementos libres. Vale destacar que la producción de los dibujos dinámicos está mediada por el uso del GeoGebra, de modo que lo importante de la prueba no es tanto la secuencia proposicional de deducciones características de una demostración formal escrita, sino la explicación razonada de lo obtenido en atención a las categorías conceptuales (Sinclair y Robutti, 2013).

Siguiendo las ideas de Acosta y Fiallo (2017), las explicaciones que conforman una prueba podrían basarse en elementos perceptivos, elementos geométricos o relaciones 
Aprendizaje de las construcciones euclidianas con GeoGebra: elementos de una actividad formativa...

detectadas en el dibujo dinámico; en algún resultado de un ejemplo cuidadosamente seleccionado, suponiendo de antemano que en cualquier otro caso se cumple el mismo resultado; en la forma de construir el dibujo dinámico o en algunos de sus pasos y acciones; en un dibujo estático del objeto esperado, sobre el cual se señalan algunas propiedades espaciales o teóricas observables (o no) que se trasladan al dibujo dinámico.

Por ejemplo, para la tarea GGB-4b, la actividad formativa puede derivar en una explicación del procedimiento de construcción del triángulo de la Figura 4 que esté guiada por la conceptualidad detrás de la herramienta Polígono. En concreto, los participantes podrían afirmar que los pasos 1 y 2 del procedimiento de construcción responden a la necesidad de conocer los vértices faltantes del triángulo ( $P$ es un objeto dado en la tarea) que se requieren para aplicar la herramienta. Además de ello, el motivo de las acciones en cada paso puede responder tanto a la necesidad de localizar los puntos $E$ y $F$ a una distancia $m$ y $n$, respectivamente de $\mathrm{P}$, como al hecho de que el ángulo $\measuredangle P$ del triángulo sea recto.

En estas explicaciones, la definición de circunferencia y la relación de perpendicularidad entre rectas resultan fundamentales. Para el ejemplo, las medidas de $\overline{P E}, \overline{P F}$ y $\Varangle P$ constituyen esas características del dibujo que han de mantenerse invariantes tras la prueba del arrastre.

\section{Interacciones}

Ya que el aprendizaje del SACEG es un fenómeno social, las interacciones entre los futuros profesores y el formador durante la resolución de las tareas de construcción euclidiana con GeoGebra constituyen un aspecto crucial del diseño de la actividad formativa. La aplicación de cada tarea implica el despliegue de un ciclo de trabajo colaborativo que está inspirado en los momentos de la actividad del aula de matemáticas de Radford (2017b). La Figura 5 muestra los cuatro momentos que conforman el ciclo de resolución de una tarea de construcción euclidiana con GeoGebra; momentos que se ajustan al tiempo de duración de cada encuentro, el número de tareas atendidas y las características del espacio físico. 
Figura 5. Ciclo de resolución de tareas de construcción euclidianas con GeoGebra

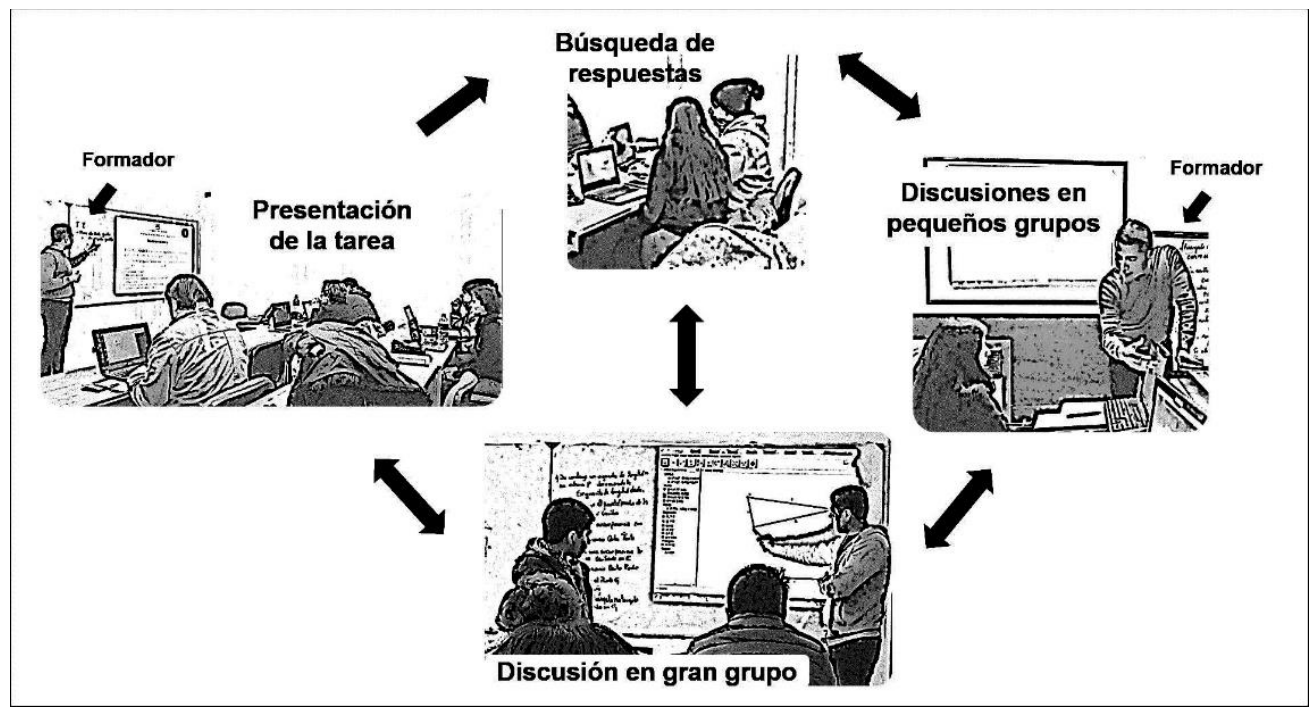

Fuente: Autores

En el momento de presentación de la tarea, el formador muestra y explica la tarea a los futuros profesores, procurando que ellos entiendan lo que deben hacer. En ocasiones, será necesario despejar aquellas dudas iniciales que puedan interferir en el desarrollo del siguiente momento. Durante la búsqueda de respuestas, los futuros profesores trabajan de manera independiente o en pequeños equipos (según las condiciones del encuentro) para producir respuestas posibles a la tarea presentada.

Para el momento de discusiones en pequeños grupos, el formador se esfuerza por reconocer las oportunidades de aprendizaje del SACEG en los modos de resolución de la tarea que surgen durante un momento de la búsqueda, identificando las respuestas que merecen ser discutidas con todo el grupo y los signos de incomprensión o frustración que impiden el avance hacia la obra común. Al centrar la atención en estas cuestiones se aumentan las posibilidades de alcanzar las metas de la formación, ya que las respuestas detectadas por el formador se convierten en la materia prima para el siguiente momento del ciclo: un momento de producción conjunta. Durante las discusiones en pequeños grupos, el formador ofrece la debida retroalimentación, responde las dudas que van surgiendo, hace preguntas y estimula la búsqueda de respuestas.

Tras decidirse las respuestas que serán compartidas y el orden de intervención de quienes las producen, se pone en marcha la discusión en gran grupo. En este momento el formador pide intencionalmente a los futuros profesores que presenten sus obras (completas o inconclusas) al 
resto del grupo, promoviendo el diálogo sobre ideas geométricas importantes (Stein et al., 2008) para la situación de resolución en que se encuentren. Normalmente, este momento se desarrolla en dos instantes. El primer instante es de reconocimiento de las condiciones de la producción de la respuesta que es compartida. En este instante el formado intenta que los futuros profesores reconozcan las potencialidades y limitaciones ${ }^{8}$ de la construcción que se les presenta, para lo cual se podría optar por solicitar a quien(es) la comparte(n) que reproduzcan y describan el procedimiento de construcción en la hoja de trabajo, desde la primera acción realizada.

El segundo instante es de modificación de la respuesta. En este instante el formador invita a los presentes a unir fuerzas y actuar responsablemente en pro de la transformación de las condiciones iniciales de producción de la respuesta compartida, lo que implica ofrecer la ayuda necesaria para la superación de las limitaciones de la construcción. Este acto demanda la movilización del SACEG para el caso de la tarea que se trate, de manera que los esfuerzos mancomunados favorezcan la toma de consciencia de aquello que necesita ser modificado, ya sea en la construcción misma (el dibujo dinámico) o en la forma de comunicar y/o probar la consistencia del procedimiento de construcción realizado. De esta manera, la respuesta inicial se transforma en la obra común de la actividad formativa.

Lograr esto requiere que los futuros profesores se sientan desafiados a actuar, para lo cual el formador puede recurrir al empleo de preguntas específicas, tales como: ¿encuentras alguna diferencia importante entre esta respuesta y la tuya? ¿Dónde está el problema en la respuesta? ¿De qué manera puedes actuar como profesor para lograr que tu compañero reconozca este problema y lo supere?

El rol que desempeña el formador en los momentos antes descritos se corresponde con la de un rol ético (Radford, 2020), en el sentido de reconocerse como parte consustancial de la actividad formativa que media y actualiza el SACEG. En otras palabras, el formador actúa éticamente en la actividad al asumirse dentro de una conciencia colectiva en la búsqueda de la obra común, problematizando la relación de los futuros profesores con el SACEG puesto en movimiento, allanando el camino hacia el logro de las metas de la formación y aportando a la

\footnotetext{
${ }^{8}$ Para conocer algunas de las limitaciones que pudieran observarse en las respuestas de futuros profesores a las tareas de construcción elaboradas, se decidió aplicarlas en 2019 al curso de Didáctica de la Matemática IV, perteneciente a la carrera de Pedagogía en Matemática y Computación de la Universidad de Los Lagos (Chile). La matrícula del curso fue de once estudiantes en el último año de su carrera universitaria.
} 
labor conjunta sus formas idiosincráticas de expresión, acción y pensamiento como un profesor de matemáticas más, dentro de un marco de actuación profesional responsable.

\section{Despliegue de una actividad formativa}

El despliegue de una actividad formativa comprende la realización en la práctica concreta de un ciclo de resolución de tareas de construcción euclidiana con GeoGebra (Figura 5). Por tanto, se espera que tal actividad ocurra tantas veces como tareas de este tipo han sido elaboradas. Para hacer operativo este diseño, se asume que la resolución de las tareas de construcción es producida en encuentros con los futuros profesores, cuya duración oscila entre 90 y 120 minutos cada uno, según las condiciones del contexto de la investigación.

En condiciones ideales, se requieren 10 encuentros para aplicar las tareas (Tabla 2), a los que se anteponen dos encuentros destinados a discutir sobre la resolución de tareas de construcción geométrica con software dinámico y la elaboración de narrativas como herramienta de formación profesional. Estos primeros encuentros tienen el propósito de crear condiciones para la buena marcha de la actividad formativa.

Tabla 2. Aplicación de las tareas por encuentros

\begin{tabular}{lcccccccccc}
\hline Encuentro & $\mathbf{1}$ & $\mathbf{2}$ & $\mathbf{3}$ & $\mathbf{4}$ & $\mathbf{5}$ & $\mathbf{6}$ & $\mathbf{7}$ & $\mathbf{8}$ & $\mathbf{9}$ & $\mathbf{1 0}$ \\
\hline Tarea(s) & GGB-1 & GGB-3a & GGB-4a & GGB-4b & GGB-5a & GGB-5b & GGB-5c & GGB-6a & GGB-6c & GGB-6e \\
& GGB-2 & GGB-3b & GGB-4b & (Caso 2) & GGB-5b & (Des. 1) & (Des. 2) & GGB-6b & GGB-6d & \\
& & & (Caso 1) & GGB-4c & & GGB-5c & GGB-5d & & & \\
\hline
\end{tabular}

Fuente: Autores

\section{Consideraciones finales}

Este trabajo presenta una caracterización de una actividad formativa que tiene por objeto el encuentro progresivo y crítico de futuros profesores de matemáticas con el saber docente acerca de las construcciones euclidianas con GeoGebra (SACEG), desde lo disciplinario. El diseño de la actividad formativa responde a la necesidad de los autores de investigar el aprendizaje del SACEG con grupos de estudiantes de carreras de Pedagogía en Matemáticas de Chile que se encuentran próximos a egresar. La decisión de focalizar la atención en la actividad formativa está justificada por la elección de una perspectiva histórico-cultural particular del aprendizaje humano (la TO) que ofrece los insumos para una conceptualización del aprendizaje del SACEG en la formación inicial de profesores de matemáticas, como procesos de objetivación. 
Aprendizaje de las construcciones euclidianas con GeoGebra: elementos de una actividad formativa...

Considerar el aprendizaje del SACEG como procesos de objetivación ha requerido de una reflexión profunda sobre la naturaleza y distintas fuentes de conocimiento que nutren el saber acerca de las construcciones euclidianas con GeoGebra. Haciendo énfasis en la vertiente disciplinaria, ha sido posible identificar y describir el saber docente que se pone de manifiesto durante la resolución de tareas de construcción euclidianas con GeoGebra, teniendo como referencia algunos problemas de construcción con regla y compás en los Elementos de Euclides que han tenido cierta trascendencia histórica (Iglesias y Ortiz, 2018). Estos dos aspectos del artículo (las ideas de aprendizaje como objetivación y SACEG como un saber docente) constituyen parte fundamental del entramado de supuestos y afirmaciones teóricas que han permitido a los autores tomar decisiones particulares en cuanto a la actividad formativa y su implementación, otorgando a su diseño un lugar privilegiado en el desarrollo de la investigación doctoral (Cai et al., 2019).

Las características del diseño permiten valorar la actividad formativa que se propone como un espacio social de toma de conciencia crítica del SACEG desde su vertiente disciplinaria, sin descuidar el sentido que se atribuye a su enseñanza. Entre las potencialidades de la actividad formativa es que su diseño posibilita que ésta pueda integrarse parcial o totalmente a la dinámica de funcionamiento de las carreras de Pedagogía en Matemáticas del país, ya sea como curso optativo, unidad de aprendizaje o actividad de mejoramiento. Finalmente, el camino iniciado permite tener una visión general de las implicaciones del diseño de entornos de aprendizaje que tenga como base una perspectiva histórico-cultural del sujeto docente y de las relaciones que este sujeto establece con el mundo de la enseñanza desde la propia universidad.

\section{Referencias}

Acosta, M. y Fiallo, J. (2007). Enseñando geometría con tecnología digital: una propuesta desde la teoría de las situaciones didácticas. Cooperativa Editorial Magisterio.

Arzarello, F., Olivero, F., Paola, D. y Robutti, O. (2002). A cognitive analysis of dragging practices in Cabri environments. ZDM, 34(3), 66-72.

Boyer, C. (1987). Historia de la matemática. Alianza Editorial.

Cai, J., Morris, A., Hohensee, C., Hwang, S., Robinson, V., Cirillo, M., Kramer, S. L. y Hiebert, J. (2019). Theoretical framing as justifying. Journal for Research in Mathematics Education, 50(3), 218-224. 
Depaepe, F., Verschaffel, L. y Kelchtermans G. (2013). Pedagogical content knowledge: A systematic review of the way in which the concept has pervaded mathematics educational research. Teaching and Teacher Education, 34, 12-25.

De Villiers, M. (1998). An alternative approach to proof in dynamic geometry. En R. Lehrer y D. Chazan (Eds.), Designing learning environments for developing understanding of geometry and space (pp. 369-393). Hillsdale: Lawrence Erlbaum.

Euclides (1991). Elementos (Libros I-IV). Editorial Gredos.

Euclides (1994). Elementos (Libros V-IX). Editorial Gredos.

Fiallo, J. (2011). Estudio del proceso de demostración en el aprendizaje de las razones trigonométricas en un ambiente de Geometría Dinámica (Tesis doctoral). Universidad de Valencia.

Fiorentini, D. (2005). A formação matemática e didático-pedagógica nas disciplinas da licenciatura em matemática. Revista de Educação PUC-Campinas, 18, 107-115.

Fischbach. F. (2015). Philosophies de Marx (Moments Philosophiques). Vrin.

Iglesias, M. y Ortiz, J. (2018). Usos del software de geometría dinámica en la formación inicial de profesores de matemáticas. Matemáticas, Educación y Sociedad, 1(2), 21-35.

Krainer, K. y Llinares, S. (2010). Mathematics teacher education. En P. Peterson, E. Baker y B. McGaw (Eds.), International Encyclopedia of Education (pp. 702-705). Oxford, UK: Elsevier.

Laborde, C. (1997). Cabri Geómetra o una nueva relación con la geometría. En L. Puig (Ed.), Investigar y enseñar. Variedades de la educación matemática (p. 33-48). Bogotá: Una Empresa Docente.

Laborde, C., Kynigos, C., Hollebrands, K., y Sträßer, R. (2006). Teaching and learning geometry with technology. En A. Gutiérrez y P. Boero (Eds.), Handbook of research on the psychology of mathematics education: Past, present and future (pp. 275-304). Rotterdam: Sense Publishers.

Leontiev, A. (1978). Actividad, conciencia y personalidad. Ediciones Ciencias del Hombre.

Llinares, S. (2004). La generación y uso de instrumentos para la práctica de enseñar matemáticas en educación primaria. UNO. Revista de Didáctica de la Matemática, 36, 93-115.

Llinares, S. (2019a). Tools and Ways of Thinking in Mathematics Teacher Education: An Introduction. En S. Llinares y O. Chapman (Eds.), International Handbook of Mathematics Teacher Education: Volume 2 (pp. 1-19). Brill Sense.

Llinares, S., Valls, J y Roig, A. I. (2008). Aprendizaje y diseño de entornos de aprendizaje basado en videos en los programas de formación de profesores de matemáticas. Educación Matemática, 20(3), 31-54.

Moise, E. E. y Downs, F. L. (1966). Geometría moderna. Addison-Wesley Publishing Company.

Neubrand, M. (2018). Conceptualizations of professional knowledge for teachers of mathematics. ZDM, 50(4), 601-612. 
Aprendizaje de las construcciones euclidianas con GeoGebra: elementos de una actividad formativa...

Radford, L. (2006). Elementos de una teoría cultural de la objetivación. Revista Latinoamericana de Matemática Educativa, Número Especial, 103-129.

Radford, L. (2013). Three key Concepts of the theory of objectification: Knowledge, knowing, and learning. Journal of Research in Mathematics Education, 2(1), 7-44.

Radford, L. (2014a). De la teoría de la objetivación. Revista Latinoamericana de Etnomatemática, 7(2), 132-150.

Radford, L. (2014b). On the role of representations and artefacts in knowing and learning. Educational Studies in Mathematics, 85, 405-422.

Radford, L (2017a). Saber y conocimiento desde la perspectiva de la teoría de la objetivación. En B. D’ Amore y L. Radford (Eds.), Enseñanza y aprendizaje de las matemáticas: problemas semióticos, epistemológicos y culturales (pp. 97-114). Bogotá: Universidad Distrital Francisco José de Caldas.

Radford, L. (2017b). Aprendizaje desde la perspectiva de la teoría de la objetivación. En B. D' Amore y L. Radford (Eds.), Enseñanza y aprendizaje de las matemáticas: problemas semióticos, epistemológicos y culturales (pp. 115-136). Bogotá: Universidad Distrital Francisco José de Caldas.

Radford, L. (2018). Pautas para repensar el sujeto y el objeto desde una epistemología de solidaridad, en tiempos de una educación para el mercado y el consumo. En A. Ávila (Coord.), Rutas de la Educación Matemática (pp. 89-105). México D.F.: SOMIDEM.

Radford, L. (2020). Un recorrido a través de la teoría de la objetivación. En S. Takeco Gobara y L. Radford (Eds.), Teoria da Objetivação: Fundamentos e aplicações para o ensino e aprendizagem de ciências e matemática (pp. 15-42). São Paulo: Livraria da Física.

Radford, L., Edwards, L. y Arzarello, F. (2009). Introduction: Beyond words. Educational Studies in Mathematics, 70(2), 91-95.

Reverand, E. (2004). Una aproximación a la teoría de la actividad. En D. Mora, A. Rivera, E. Reverand, W. Beyer, W. Serrano, O. Brito y C. Torres (Eds.), Tópicos en Educación Matemática (pp. 151-181). Caracas: GIDEM.

Rey, J. y Babini, J. (1985). Historia de la matemática (vol. 1). Gedisa.

Ríbnikov, K. (1987). Historia de las matemáticas. Editorial Mir.

Scriba, C. y Schreiber, P. (2015). 5000 Years of Geometry. Mathematics in History and Culture. Springer.

Simson, R. (2014). Los seis primeros libros, y el undécimo y duodécimo de los Elementos de Euclides. Editorial Maxtor.

Sinclair, N. y Robutti, O. (2013). Technology and the role of proof: the case of dynamic geometry. In A. Bishop, K. Clement, C. Keitel, J. Kilpatrick, \& F. Leung (Eds.), Third international handbook of mathematics education (pp. 571-596). Berlin: Springer.

Stein, M., Engle, R., Smith, M. y Hughes, E. (2008). Orchestrating productive mathematical discussions: five practices for helping teachers move beyond show and tell. Mathematical Thinking and Learning, 10, 313-340.

Tardif, M. (2002). Los saberes del docente y su desarrollo profesional. Narcea Editores. 
Tirosh, D. \& Wood, T. (2008). Tools and processes in mathematics teacher education: an introduction. En D. Tirosh y T. Wood (Eds.), The International Handbook of Mathematics Teacher Education (pp. 1-14). Rotterdam: Sense Publishers.

Tosca, T. (1757). Compendio Mathematico. Tomo I: en que se contienen todas las materias más principales de las ciencias que tratan de la cantidad. Imprenta de Antonio Bordazar.

Valente, W. R. (2017). A matemática $a$ ensinar e a matemática para ensinar: os saberes para a formação do educador matemático. En R. Hofstetter y W. Valente (Orgs.). Saberes em (trans)formação: tema central da formação de professores (pp. 201-229). São Paulo: LF Editorial.

Vega-Reñon, L. (1990). La trama de la demostración. Los griegos y la razón tejedora de pruebas. Alianza editorial.

Watson, A. y Ohtani, M. (2015). Themes and issues in mathematics education concerning task design: Editorial introduction. En A. Watson y M. Ohtani (Eds.), Task design in mathematics education, an ICMI Study 22 (pp. 3-15). NY: Springer.

Xavier, L. N. A. (2014). A construção social e histórica da profissão docente: uma síntese necessária. Revista Brasileira de Educação, 19(59), p. 827-849.

Autores

Juan Luis Prieto González

Estudiante del Programa de Doctorado en Educación Matemática de la Universidad de los Lagos (Chile); Máster en Nuevas Tecnologías Aplicadas a la Educación (Universidad Autónoma de Barcelona, Universidad de Alicante, Universidad Carlos III de Madrid, España, 2009); Diplomatura en Estudios Avanzados en la línea de Didáctica de las Matemáticas (Universidad de Alicante, 2008, España); Coordinador General de la Asociación Aprender en Red (http://aprenderenred.com.ve/). Tesorero de la Asociación Venezolana de Educación Matemática (AsoVeMat). Email: juanl.prietog@gmail.com

Elizabeth-H- Arredondo

Doctora en Ciencias, Especialidad en Matemática Educativa, por parte del CINVESTAV-IPN, México. Académica del Departamento de Ciencias Exactas de la Universidad de Los Lagos, Osorno, Chile. Catedrática del Programa de Postgrado de Magister y Doctorado en Educación Matemática de la Universidad de Los Lagos. E-mail: elizabeth.hernandez@ulagos.cl 